FORMATION Formation emploi

Revue française de sciences sociales

130 | Avril-Juin 2015

L'essor des compétences non académiques

\title{
La gestion paradoxale des compétences de service
}

The paradoxical management of service skills

Das paradoxale Management von Dienstleistungskompetenzen

¿Hay que ser competente para poder desarrollar las propias competencias? El

ejemplo de la selección para entrar en Master 2

Damien Collard, Jean-Baptiste Suquet et Nathalie Raulet-Croset

\section{(2) OpenEdition}

Journals

Édition électronique

URL : http://journals.openedition.org/formationemploi/4410

DOI : 10.4000/formationemploi.4410

ISSN : 2107-0946

Éditeur

La Documentation française

Édition imprimée

Date de publication : 10 juin 2015

Pagination : 49-67

ISSN : 0759-6340

Référence électronique

Damien Collard, Jean-Baptiste Suquet et Nathalie Raulet-Croset, « La gestion paradoxale des compétences de service », Formation emploi [En ligne], 130 | Avril-Juin 2015, mis en ligne le 08 juin 2017, consulté le 30 octobre 2020. URL : http://journals.openedition.org/formationemploi/4410 ; DOI : https://doi.org/10.4000/formationemploi.4410 


\title{
La gestion paradoxale des compétences de service
}

\author{
Damien Collard
}

Maître de conférences à l'université de Franche-Comté, chercheur au Centre de recherche en gestion des organisations (CREGO) de l'université de Bourgogne

JEAN-BAPTISTE SUQUET Professeur associé à Neoma Business School, membre du Centre de recherche sur le management des clients (CMAC) et chercheur associé à I'Institut de recherche en gestion (IRG) de l'université de Paris-Est Marne la Vallée

Nathalie Raulet-Croset Maître de conférences à I'IAE (Institut d'Administration des Entreprises) de l'université Paris 1, chercheure au Centre de recherche en gestion (CRG) de l'Ecole Polytechnique et chercheure associée au Groupe de Recherche en Gestion des Organisations (GREGOR) de I'IAE de Paris

Résumé

\section{La gestion paradoxale des compétences de service}

Cet article s'intéresse aux compétences de service mobilisées par les agents au contact de la clientèle et qui sont la plupart du temps tacites. À ce titre, s'interroger sur la reconnaissance de ces compétences et sur leur développement constitue un enjeu de gestion pour les organisations. C'est d'autant plus important que de nombreux métiers de service allient faible qualification et fortes compétences situées.

Mots clés : Compétence, compétence relationnelle, savoir professionnel, FPC en entreprise

Abstract

The paradoxical management of service skills

This paper focuses on service skills which are mobilized by front office agents when they are involved with customers. These skills are most of the time tacit. We consider that the way in which firms recognize these skills and contribute to their development is an important management issue. It is all the more important that a lot of jobs combine poor levels of qualification and highly situated skills.

Keywords: Skill, relationship skills, professionnal knowledge, CVT in companies Journal of Economic Literature: J 24, M 53, M 54

Traduction : Auteurs 
La plupart des managers opérationnels et des spécialistes RH (ressources humaines) estiment que ce qui importe pour prévoir le comportement professionnel futur, ce ne sont pas tant les diplômes que les "qualités relationnelles", les «compétences sociales", ou encore les "savoir-être" (Bellier, 2000). Autant de compétences " non académiques " ${ }^{1}$ qui s'opposent aux savoirs disciplinaires et à la " compétence scolairement garantie " (Bourdieu, 1998). McClelland (1961) considère ainsi que ni les diplômes ni les tests d'aptitudes ne sont de bons indicateurs de la réussite professionnelle (Mitrani et al., 1992), contrairement aux compétences comportementales. Ces dernières seraient transférables d'une situation professionnelle à une autre et constitueraient un gage d'employabilité, d'où l'importance qu'elles revêtent pour la gestion des ressources humaines (GRH). D'autant plus que l'on attend aujourd'hui des salariés qu'ils sachent collaborer, travailler en réseau, partager des connaissances...

Ces compétences deviennent encore plus cruciales en raison de la montée en puissance du secteur des services. En effet, de plus en plus de salariés sont en contact direct avec un " client " (voir par exemple MacDonald \&Merrill, 2009) et doivent, dès lors, mobiliser des compétences adaptées (Alis, 1994 ; 1998). Si certaines d'entre elles constituent des compétences comportementales très générales, d'autres sont a contrario très spécifiques, tacites et ancrées dans des situations particulières.

Nous voudrions ici nous focaliser sur les "compétences de service " qui sont mobilisées dans le contact avec le client pour résoudre des problèmes concrets. Elles diffèrent grandement des compétences comportementales définies par McClelland et n'existent que dans l'action et en situation. Ce sont, pour les agents au contact du public, des capacités à faire en situation (pour les contrôleurs de bus par exemple, varier leur intonation entre la fermeté et l'empathie, au fur et à mesure du contrôle et des réactions des voyageurs) - et, en ce sens, elles sont une source de performance car elles améliorent la relation entre une entreprise et ses clients.

À ce titre, s’interroger sur la reconnaissance de ces compétences et sur leur développement constitue un enjeu de gestion pour les organisations, dans la mesure où elles sont inséparables des situations d'interaction. C'est d'autant plus pertinent que nombre de métiers de service allient faible qualification et fortes compétences situées (on pense notamment à la capacité des hôtes de caisse à gérer le flux des clients). La difficulté à rabattre ces compétences sur des qualifications les rend insaisissables et difficiles à objectiver - ce qui est à la fois consubstantiel à la notion de compétence, mais en même temps particulièrement marqué dans le cas des compétences de service (s'agissant de l'intonation par exemple). Ces dernières constituent donc, d'une certaine façon, un cas extrême, notamment intéressant pour interroger les limites des outils classiques de gestion de ces compétences, comme la GPEC (gestion prévisionnelle des emplois et des compétences).

1.Nous renvoyons le lecteur à l'article de Marie Duru-Bellat dans le présent numéro pour une plus ample présentation de ce concept. 
Ainsi, la gestion classique des compétences, qui est souvent par essence a-contextuelle, estelle véritablement adaptée au caractère situé des compétences de service ? Pour mettre au jour des compétences de service spécifiques et réfléchir à ces questions, nous nous appuierons sur deux enquêtes, menées au sein de la SNCF, auprès des agents en contact avec la clientèle. Après une revue de littérature sur les compétences de service, nous présenterons notre terrain, la méthodologie employée et quelques compétences de gestion de la relation repérées lors de nos deux enquêtes, puis nous nous interrogerons sur la reconnaissance et le développement de telles compétences.

\section{Les compétences de service : des compétences plurielles et situées?}

Des travaux d'analyse des situations de relation de service, en sociologie, en ergonomie et en sciences du langage principalement, ont permis depuis longtemps de mettre en évidence "l'extension et la variété " des registres de compétences des agents au contact du public (Strobel, 1994) (première partie). Celles-ci se caractérisent par leur caractère tout à la fois routinier et dépendant de contextes spécifiques. Elles sont donc difficiles à identifier, à décrire et à reconnaître (seconde partie).

\subsection{Des compétences plurielles}

Les compétences de service sont le plus souvent circonscrites au registre du relationnel client. Bien qu'elles soient également techniques, la littérature a surtout contribué à rendre visible tout un registre relationnel et organisationnel de compétences.

\subsubsection{Les compétences techniques}

Les compétences de service sont "indissociablement des compétences techniques et de service ", puisqu'il s'agit dans les services "d'une double activité : la gestion de l'intervention sur l'objet et la gestion de la relation de service " (Mayen, 2005). On peut distinguer en premier lieu un ensemble de compétences dites "techniques» qui consistent à accomplir des actes, comme par exemple "réaliser des repas, exercer des interventions chirurgicales, savoir conduire un bus, etc. "(Ughetto, 2002, p. 36). La compétence technique, selon Marie-Christine Combes, tend à devenir " de plus en plus sophistiquée " (Commissariat Général au Plan, 2001). Les changements technologiques, par exemple le développement des technologies numériques dans les banques (Värlander \& Julien, 2010), confortent l'enjeu de maîtrise de compétences techniques. Ces dernières sont généralement aisées à identifier, à circonscrire et à gérer. 


\subsubsection{Les compétences de gestion de l'interaction}

Un autre ensemble de compétences de service renvoie à des «savoirs de gestion de l'interaction" (Caroly \& Trompette, 2006). En premier lieu, faire preuve de pédagogie, ou encore avoir une "aptitude à se mettre à la place de l'autre " (Borzeix, 1995). Se représenter le point de vue des clients n'amène cependant pas nécessairement à l'adopter. L'agent doit en effet savoir " exercer du pouvoir dans [la] relation" (Gadrey, 1994a). Cette tension inhérente à la coproduction du service conduit à d'inévitables arbitrages, notamment entre les attentes des clients et les règles de l'organisation du service (Ughetto, 2002), parfois à l'origine de conflits, et la capacité à pacifier la relation a son importance. Les rencontres avec le public nécessitent ainsi une "gestion des émotions" de la part de l'agent (Boujut, 2005 ; Bernard, 2007). Le travail émotionnel et les compétences qu'il suppose ont d'ailleurs fait l'objet de nombreux débats (Payne, 2009 ; Korczynski, 2005) suite aux travaux fondateurs de Hochschild (1983). Chacune de ces compétences est à mettre en relation avec une compétence plus générale, qui les intègre et les articule dans l'interaction. Elle consiste à élaborer et adapter des scripts qui permettent d'écourter l'interaction en automatisant certains aspects de cette dernière (Cosnier et al., 1992). Ainsi, face à certaines objections récurrentes, les agents au contact peuvent répondre aux clients en mobilisant des enchâ̂nements d'arguments déjà éprouvés.

\subsubsection{Les compétences communicationnelles}

Les compétences communicationnelles sont largement mises en lumière dans les travaux sur le travail de service, notamment s'agissant de la communication non-verbale, "gestes, mimiques et voix" (Grosjean, 1995). Certains auteurs signalent également des compétences esthétiques, autrement dit les dispositions incorporées des agents à transmettre une image satisfaisante, en cohérence avec l'image souhaitée par l'organisation (Bailly et Léné, 2013).

Plus généralement, les travaux fondateurs d'Erving Goffman (1968) et ceux qui les ont suivis (Joseph et al., 1995) attribuent aux interactants une compétence civile, c'est-à-dire une capacité à suivre des rituels de civilité quotidiens. L'agent au contact peut, enfin, avoir à adapter ses pratiques de communication. Michel Dartevelle, évoquant les contrôleurs de la RATP (régie autonome des transports parisiens), souligne ainsi leur " mobilité langagière, c'est-à-dire [leur] capacité à changer de registre selon la situation et le moment de l'interaction " (Dartevelle, 1992).

\subsubsection{Les compétences cognitives}

La littérature insiste sur le fait que les agents au contact du public sont confrontés à des " situations incertaines ", qui leur demandent des " capacités de jugement et d'interprétation des situations" (Havard, 2006). Ainsi, la réalisation même du travail passe par une catégorisation des clients (Weller, 1997), compétence essentielle d'un agent pour «transforme[r] son problème ou ses questions en cas (typification)»(Gadrey, 1994b, p. 385). Gadrey souligne 
les routines mobilisées par les agents, qui sont "appuyées selon les cas sur des dossiers, formulaires, ou sur des guides méthodologiques plus complexes et plus ouverts" (Ibid.).

\subsubsection{Les compétences d'organisation}

Parce que la relation de service implique "la production d'une succession d'agencements organisationnels singuliers" (Caroly et al., 2006, p. 11), faisant intervenir une multiplicité d'acteurs, de règles, d'outils, etc., les recherches mettent en évidence une compétence d'organisation. Celle-ci renvoie à une "compétence à collecter l'information nécessaire ", ainsi qu'une capacité "à mobiliser d'autres compétences que la sienne propre pour aboutir à une solution " (Commissariat Général au Plan, 2001, p. 91). Dans ce registre, Caroly et Trompette (2006) évoquent, d'une part, les " compétences de coordination » et, d'autre part, les " compétences d'orchestration" pour décrire des capacités "d'ordonnancement des tâches, d'anticipation et de prévention des dysfonctionnements possibles" (Ughetto, 2002, p. 40).

\subsection{Des compétences tacites difficiles à objectiver}

On a pu voir combien les compétences de service sont de l'ordre du micro-social et s'avèrent largement tacites. Elles sont d'autant plus difficiles à repérer, à décrire, et donc à reconnaître.

La littérature souligne la difficulté à reconnaître des compétences «soft » et l'enjeu que cela représente pour leur développement (Korczynski, 2005). Les obstacles rencontrés par les gestionnaires sont, d'une part, l'incapacité à distinguer la compétence de la caractéristique personnelle (Gamble, 2006 ; Payne, 2009), vue ici comme naturelle, innée (Bailly et Léné, 2013) et, d'autre part, le besoin de mesurer des compétences qui ne s'y prêtent pas (Korczynski, 2005). Inversement, certains auteurs incitent prudemment à ne pas verser dans une reconnaissance trop large qui amènerait paradoxalement à voir des compétences partout, et donc à perdre de vue l'intérêt du concept même de compétence (Payne, 2009). La question sous-jacente à ces débats est de comprendre comment les compétences de service apparaissent dans les organisations. En réponse à cette question, nous mettrons tout d'abord en évidence le rôle éminent joué par le collectif de travail, avant de souligner l'ancrage de ces compétences dans des situations particulières.

\subsubsection{Le rôle clé du collectif de travail}

De nombreux auteurs soulignent la dimension collective de ces compétences de service. Ainsi, Marie-Christine Combes soutient que "les compétences individuelles ne sont plus compréhensibles indépendamment des compétences collectives" (Combes, 2002, p. 12). Selon Christelle Havard (2006, p. 5), "ces compétences client ne sont pas seulement individuelles, elles sont aussi portées par le groupe de travail (...), voire par l'organisation ".

Cependant, la compétence de service ne procède pas d'un collectif homogène et abstrait ; elle réside dans une division du travail propre à l'équipe : la compétence est distribuée 
dans l'équipe à l'occasion de la réalisation du travail (Borzeix et al., 1999). Il y a ici « l'idée que l'organisation collective du travail peut constituer un espace de développement des compétences individuelles" (Caroly et al., 2006, p. 2). Les auteurs reviennent notamment sur l'importance des échanges entre membres du collectif de travail : "Le principal outil de diffusion des compétences et des connaissances (...) est la discussion" (Honoré, 2002). Damien Collard et Nathalie Raulet-Croset (2006) soulignent le rôle central de la parole au sein d'un collectif de travail pour l'articulation des compétences tacites. Enfin, certains auteurs en appellent à délaisser une approche finalement peu explicite de la compétence collective, au profit d'une véritable analyse de celle-ci et du contexte organisationnel dans lequel elle se déploie (Krohmer, 2006).

\subsubsection{L'indexation des compétences sur la situation}

Évoquer le contexte organisationnel permet de rappeler que les compétences sont mises en œuvre en situation, la situation étant à la fois structurée par une organisation et émergente (Collard et al., 2013).

Structurée, d'une part, puisque l'action en situation suppose l'intervention d'acteurs extérieurs à l'interaction de service. Le Breton pointe la contribution décisive des services fonctionnels ou supports (Le Breton, 1999) dans la constitution d'une compétence de service.

L'encadrement, par un travail d'argumentation, de figuration et d'évaluation, participerait également au développement des compétences (Tannery, 2001).

De plus, "les compétences collectives se cristallisent dans des règles explicites ou tacites qui sont définies et adoptées par le collectif de travail. Elles peuvent être des règles imposées par la hiérarchie [...] ou résulter de consensus établis lors de réunions collectives" (Havard, 2006, p. 6).

Enfin, l'organisation prévoit le déroulement des interactions et équipe les agents au contact du public, ce qui fait dire à Jean-Marc Weller, par exemple, que « les compétences de l'agent de bureau dépendent aussi de son guichet" (Weller, 1999).

Mais, d'autre part, la situation reste irréductiblement émergente et en partie imprévisible : ce sont des "événements qui vont solliciter la compétence des agents" (Valléry, 2004, p. 123). Il y a donc peu de sens à réifier les compétences en les désignant en dehors de la situation où elles apparaissent (Weller, 1997). D'ailleurs, c'est souvent l'organisation elle-même qui génère un certain nombre de contradictions et rend nécessaires de multiples arbitrages (Collard et al., 2013). Le caractère irréductiblement imprévisible des situations rend l'appréhension de ces compétences difficile pour les organisations.

Les démarches classiques de GPEC passent par la recension et la mise en forme des compétences de service dans des référentiels (Baroin-Loos, 2003). Mais le risque d'une formalisation est d'aller à l'encontre d'une prise en compte de la complexité des situations de travail. Sophie Beauquier a ainsi mis en évidence la focalisation d'entreprises " orientées-client» comme la RATP (régie autonome des transports parisiens) et ASSUR, sur les 
registres relationnels des métiers au contact du public : "Face à ces difficultés de description et de formalisation des nouveaux rôles et des nouvelles compétences, la direction s'est rabattue sur la notion "fourre-tout " de compétence relationnelle qui va désormais résumer les compétences attendues et qui constituerait désormais le cour de ces nouveaux métiers de la relation de service. La primauté donnée à ces compétences conduit à faire l'impasse sur le contenu réel de ces nouveaux métiers qualifiés, à tort, de relationnels car ils mobilisent au moins autant des compétences techniques. " (Beauquier, 2003, p. 149)

Retenons donc que le travail des agents de contact repose sur la mobilisation de capacités relativement insaisissables et difficiles à objectiver, du fait en particulier de leur caractère co-construit et situé. Venons-en maintenant aux compétences de service des agents de la SNCF en contact avec la clientèle.

\section{Encadré 1 : Méthodologie}

Pour décrire les compétences de ces agents, nous nous appuierons sur deux enquêtes portant sur la relation de service, menées à quelques années d'intervalle au sein de la SNCF. La première, qui a été réalisée dans le cadre d'une thèse de doctorat effectuée sous convention CIFRE (convention industrielle de formation à la recherche) à la SNCF (Collard, 2003), nous a permis d'identifier les compétences mises en œuvre par les agents d'ambiance qui avaient été embauchés sous statut emploi-jeune par l'entreprise $\left(^{*}\right)$. La seconde résulte d'un contrat de recherche passé avec le Centre de recherche en gestion de l'École polytechnique qui portait sur l'identification des « compétences implicites de service » mobilisées par les agents en contact avec la clientèle de la SNCF ${ }^{* *}$ ) (Borzeix et al., 2008).

Lors de la première enquête, le chercheur a adopté une démarche de type ethnographique. Intégré en tant que chargé de mission au sein de la Direction régionale des ressources humaines de Paris Sud-Est, il a endossé différents rôles. II a commencé par analyser le travail réalisé par les équipes d'agents d'ambiance travaillant dans les gares rattachées à l'Établissement d'exploitation de Melun. Il a notamment procédé par observation participante sur l'une des gares les plus « sensibles » de cet établissement - celle d'Evry-Courcouronnes - en intégrant l'équipe des agents d'ambiance pendant cinq semaines (dans le courant de l'année 1999). Ensuite, il a participé à la mise en place de formations à destination des agents et animé un groupe de travail intitulé " veille professionnelle et enrichissement des tâches », composé d'encadrants d'équipes d'agents d'ambiance. Sa mission consistait à réfléchir au contenu des tâches qui avaient été confiées aux agents d'ambiance en vue de les enrichir.

Lors de la seconde enquête, deux des auteurs ont suivi des agents au contact du client. Ils ont enregistré (via un dictaphone) des interactions agents-clients, assorties parfois d'un " retour » des agents. Des entretiens avec des managers ont également été menés.

Sur les dix-huit mois de l'étude, ils ont pu collecter 240 observations d'interactions et réaliser 45 interviews approfondies (d'une durée moyenne supérieure à 45 minutes). Ce matériau a été traité de manière systématique, en plusieurs étapes. 


\section{Suite encadré 1}

Lors de la première étape, ils ont « découpé » l'ensemble des enregistrements et prises de notes en un ensemble de situations, à savoir des séquences d'interactions entre un (ou plusieurs) agent(s) et des clients, pour lesquelles ils ont identifié un début et une fin, ainsi qu'une certaine continuité de l'action. Parmi ces situations, certaines renvoyaient à des routines de traitement relativement simples ; d'autres leur ont semblé présenter un caractère plus complexe, nécessitant de fait un engagement plus important des agents. Ces dernières situations ont été analysées pour tenter de mettre à jour les compétences particulières mobilisées par les agents. Adoptant une méthode de théorisation "ancrée » (Glaser et Strauss, 1967), ils ont dégagé, à partir de l'analyse des situations répertoriées, celles susceptibles d'inclure des compétences implicites de service. Progressivement, et de façon inductive, des ensembles de situations de même nature et des compétences liées à la capacité des agents à résoudre certains problèmes ont été identifiés.

$\left(^{*}\right)$ : Il s'agissait d'étudier un « métier » en émergence et de braquer le projecteur sur un public (des jeunes peu ou pas diplômés) qui rencontrait des difficultés à s'insérer professionnellement. Le programme « Nouveaux services - Emplois jeunes » avait été lancé par le Gouvernement de Lionel Jospin en 1998. Dans le cadre de ce programme, environ 2000 jeunes avaient été recrutés par la SNCF.

$\left.{ }^{* *}\right)$ : Cette recherche a été réalisée à la demande de la Direction de l'innovation et de la recherche et de l'Université du service de la SNCF. Il s'agissait de décrire et d'analyser des compétences qui n'étaient pas répertoriées par les outils de gestion existants.

\section{Les compétences de service spécifiques des agents de la SNCF en contact avec la clientèle}

La population étudiée est celle des agents de la SNCF qui exercent un métier en contact avec les voyageurs, que cela soit pour les renseigner et les orienter (agents d'ambiance et agents d'escale), leur vendre des billets (agents de vente) ou encore contrôler la validité de leur titre de transport (contrôleurs).

Après avoir décrit quelques compétences mises en œuvre par les agents d'ambiance embauchés sous statut emploi-jeune, nous examinerons un exemple de compétence mobilisée par les agents statutaires de la SNCF, à savoir la capacité à allier fermeté et civilité.

\subsection{Les agents d'ambiance : entre compétences de médiation et compétences commerciales}

Les emplois d'agents d'ambiance n'ont perduré que le temps du programme « Nouveaux services - Emplois jeunes " et n'ont finalement pas été pérennisés par l'entreprise. Cependant, quasiment tous les jeunes qui sont passés par ce dispositif ont intégré l'entre- 
prise sur les métiers existants (pour la plupart dans la filière commerciale), grâce à l'expérience acquise en tant qu'agent d'ambiance.

L’observation participante menée auprès des agents d'ambiance de la gare d'Evry-Courcouronnes - qui étaient issus pour la plupart des cités "sensibles » avoisinantes - a révélé que ces agents s'appuyaient sur des ressources à la fois langagières, comportementales et cognitives dans le contact avec le public. Leur " clientèle " ne s'arrêtait pas aux clients SNCF, mais s'étendait à tous ceux qui occupaient l'espace de la gare.

Ces agents catégorisaient d'ailleurs de manière extrêmement fine les différents publics. Par exemple, parmi les « indésirables » de la $\mathrm{SNCF}^{2}$, les agents distinguaient les "zonards », les " toxicos ", les " psychos ", les " mythos ", les « hystériques ", les « mystiques », etc. Cette terminologie, qui leur était propre, équivalait à des mini-schèmes d'action.

Elle permettait une économie d'interprétation et l'adoption de comportements-types pertinents : être détendu ou vigilant, créer de la convivialité ou garder ses distances, plaisanter ou rester froid et laconique, dialoguer ou faire un " coup de pression »...

Les agents d'ambiance, qui inscrivaient leur action dans un double registre, professionnel et familier, avaient notamment développé des modes de résolution des conflits cohérents avec les us et coutumes en vigueur dans l'environnement local, en faisant en sorte que personne ne perde la face et en pratiquant la culture du « deal» (c'est-à-dire en fermant les yeux sur certains comportements répréhensibles pour obtenir la paix sociale sur la gare...).

Cependant, tous les agents n'avaient pas forcément développé les mêmes compétences. Ils s'étaient en effet implicitement répartis les rôles en fonction de leurs ressources et du type de problème rencontré. Ainsi, certains agents (issus des cités "sensibles ") s'étaient spécialisés dans la gestion des relations avec les "indésirables » et avaient développé de véritables compétences de médiation ${ }^{3}$, tandis que d'autres prenaient plus volontiers en charge les tâches relatives à l'information et à l'orientation des voyageurs et avaient de ce fait développé des compétences plus commerciales.

\subsection{Les agents statutaires de la SNCF au contact des voyageurs : allier fermeté et civilité}

L'enquête menée auprès des agents statutaires de la SNCF révèle une multitude de compétences de service. Celles-ci constituent des combinaisons de ressources hétérogènes

2. Ce terme renvoie à une catégorie propre à la SNCF, désignant les personnes occupant l'espace de la gare sans être pour autant des voyageurs, lesquels pouvaient se sentir dérangés par les "indésirables ».

3. Catégoriser les "indésirables ", adopter un comportement approprié à la situation, "évaluer " une ambiance, établir le contact et gagner la confiance d'un individu..., constituent autant de compétences de médiation qui n'étaient cependant pas reconnues en tant que telles par la SNCF. Ces compétences sous-tendent une connaissance intime des individus, de la gare, de ce qui s'y passe et de ce qui s'y trame, une capacité aussi à s'effacer et à se fondre dans le paysage. 
mobilisées dans l'action et dans des situations spécifiques (Le Boterf, 1994). Certaines ressources sont apportées par l'organisation (par exemple les procédures de travail et les modes opératoires), tandis que d'autres sont très contingentes aux individus (à leur personnalité, à la conception qu'ils ont de leur rôle). L'analyse des situations de travail révèle certaines tensions résultant de contraintes portées par les différents acteurs (clients, collègues, managers, agent lui-même, etc.) ou issues du contexte organisationnel. L'obligation pour l'agent de « faire face » au client est ressentie d'autant plus fortement qu'il opère dans un contexte organisationnel très cadré, dont il ne maitrise presque aucun des choix. Ainsi, pour offrir au client une réponse unique et cohérente, il doit résoudre dans l'immédiateté d'une relation de face à face, les contradictions éventuelles de l'entreprise.

Prenons un exemple concret. Il s'agit d'un agent d'escale, plus précisément d'un adjoint départ de train : celui-ci doit à la fois respecter des procédures de sécurité et renseigner les voyageurs. Dans la situation ci-dessous, intitulée par l'agent lui-même " le bras de fer ", il s'agit pour l'agent de trouver un équilibre entre une attitude empreinte de fermeté, qui renvoie à un objectif d'évitement de risques, et une attitude attentionnée, qui respecte les fondamentaux de la relation de servicé ${ }^{4}$. L'enjeu consiste à faire appliquer des règles strictes à des voyageurs, inconscients la plupart du temps des risques, comme dans la situation ci-dessous.

\section{Encadré 2 : Le « bras de fer »}

L'agent vient de procéder au départ du train. Deux jeunes voyageurs arrivent en trombe sur le quai et se précipitent vers le train.

L'agent s'interpose en adoptant une attitude de grande fermeté : "C'est terminé! On ne monte pas! » (l'agent fait barrage avec son corps et fixe les deux jeunes droit dans les yeux). Ils obtempèrent.

L'agent (calmement) : «Alors, le prochain train pour Belfort, c'est $18 \mathrm{~h} 34$ ».

L'agent (commentant la situation après coup) : «C'est vrai que quand je dis qu'on ne monte pas, on ne monte pas! Quand j'ai dit non, c'est non! (...). Ça c'est un apprentissage qui se fait sur le terrain. Le "bras de fer", c'est sur le terrain ".

Dans cet exemple, l'irruption des jeunes retardataires induit une tension forte et un risque d'accident. En prenant contact et en s'interposant, l'agent crée une situation d'interaction. Les capacités requises pour gérer cette situation renvoient à des attitudes corporelles (bras et regard) et au basculement opéré par l'agent pour passer d'un registre relationnel à un autre (du sécuritaire à l'informatif) qui désamorce d'emblée un possible conflit et modifie le sens donné à la situation. Il définit un cadre qui lui permet tout à la fois de marquer son autorité, de barrer l'accès au train, et de transformer la situation initiale en une situation

4.Les agents doivent en effet respecter des normes et des attitudes de service. 
d'information. Tout l'art de l'agent réside ici dans le fait de passer d'un registre d'action à un autre, et ce dans un temps très court. Cela demande une grande réactivité puisqu'il s'agit d'établir très rapidement un diagnostic de la situation (l'agent mobilise ici un « savoir voir »), du sang-froid (l'enjeu est de gérer les émotions d'autrui et ses propres émotions), une grande fermeté, mais également un certain doigté (puisque le but de l'agent est de recadrer la situation pour offrir aux clients une alternative). Tous ces savoir-faire, par ailleurs bien documentés dans la littérature sur les compétences de service, se combinent en situation pour former une compétence plus générale que nous avons intitulée « doser fermeté et civilité »(Collard et al., 2013).

\section{Comment reconnaître et développer 3 les compétences de service?}

Lidentification des compétences de service mises en jeu dans l'activité professionnelle devient ainsi possible dès que l'on observe les situations de travail et que l'on identifie avec les agents les tensions qui structurent ces situations.

Mais dans quelle mesure cette démarche est-elle compatible avec les approches organisationnelles classiques de la gestion des compétences, comme la sélection, la formation, ou encore la GPEC, plus adaptées à des compétences techniques et à des compétences comportementales très générales?

\subsection{La difficile inscription organisationnelle des compétences de service}

Dans les deux cas étudiés (agents d'ambiance sous statut emploi-jeune et agents statutaires de la SNCF en contact avec la clientèle), l'instrumentation gestionnaire s'est révélée inadaptée et n'a pas permis de reconnaitre pleinement les compétences développées par les agents dans leur travail.

Dans le premier cas, le système de GRH de la SNCF ne reconnait pas les compétences de médiation acquises par les agents d'ambiance. Les catégories gestionnaires et les outils de GRH utilisés en interne ont "écrasé » le travail réel et une partie des compétences mises en jeu dans l'exercice de l'activité (Collard, 2003). Il en est ainsi du livret de professionnalisation. Les compétences attendues contenues dans ce livret (cf. encadré 3), et qui servaient de support d'évaluation pour l'encadrement de proximité, étaient en effet assez éloignées des compétences acquises par les agents en situation. 


\section{Encadré 3 : Les compétences liées à la gestion de l'espace (extrait du livret de professionnalisation)}

- Alerter les services compétents en cas de panne d'un automate ;

- Alerter et intervenir éventuellement en cas de danger (objets, colis suspects) ;

- Alerter et intervenir éventuellement en cas de situations anormales ;

- Contribuer à travailler efficacement avec les partenaires ;

- Participer éventuellement à des opérations d'animation.

Les fiches de poste, quant à elles, apparaissaient lacunaires, dans la mesure où elles ne disent rien ou quasiment rien des compétences requises dans l'exercice du métier. Quant au référentiel de compétences de l'agent d'ambiance, il ne fait état ni de l'activité de médiation sociale ni des compétences de médiation.

Dans le second cas, les outils de gestion ont gommé les tensions et les dilemmes propres à l'activité des agents d'escale. La situation intitulée "le bras de fer ", évoquée précédemment, révèle en effet que les agents d'escale doivent tenir compte, dans leur travail, à la fois des règles de sécurité et des normes de service, et que les compétences de service mobilisées sont bien à la fois techniques et de service (Mayen, 2005). Ces deux types de règles peuvent évidemment entrer en tension, voire se révéler antagonistes. Mais ces tensions ne sont pas reconnues par les donneurs d'ordre. Pour les spécialistes de la sécurité, seule compte en effet l'application stricte de la réglementation. A contrario, pour ceux qui pilotent la relation de service, seul compte le respect des normes et des attitudes de service ( $c f$. encadré 4).

\section{Encadré 4 : Les « attitudes phares » définies par la Direction des gares et de l'escale en 2007}

1.) L'agent est « proactif » : il va au-devant du client et prend l'initiative du «Bonjour Monsieur », «Bonjour Madame»;

2.) Il prend poliment congé du client en utilisant la formule «Au revoir Monsieur », «Au revoir Madame »;

3.) Il porte son badge qui mentionne son prénom et la formule « à votre service » ;

4.) II explique tous les services avec pédagogie et rend le client autonome pour son prochain voyage.

Des enquêtes client mystère ont ainsi été mises en place pour vérifier la bonne application de ces normes par les agents d'escale ; il a en outre été demandé expressément aux dirigeants de proximité d'évaluer les comportements de leurs agents au regard de ces normes (Collard, 2013). L'encadrement de proximité dispose ainsi d'outils spécifiques pour évaluer des dimensions particulières de l'activité des agents d'escale, mais n'est pas à même de 
prendre en compte cette activité dans sa globalité et dans sa complexité. Il y a donc des angles morts dans l'instrumentation gestionnaire et peut-être là une impasse...

\subsection{La fausse évidence de la formation}

Plusieurs auteurs mentionnent la formation comme un moyen de développer les compétences de service. Certains considèrent même qu'une formation de qualité centrée sur la notion de service et les attitudes de service est un prérequis pour développer de telles compétences.

Pour certains, ces formations de qualité ne sont guère plus qu'un habillage rhétorique de pratiques de gestion des compétences déjà en place (Korczynski, 2005).

Par ailleurs, toutes les organisations ne semblent pas prêtes à investir dans la formation, loin s'en faut, compte tenu de la croyance répandue dans le caractère inné des compétences de service (Bailly et Léné, 2013).

Enfin, le caractère tacite de ces compétences est difficilement pris en charge par les acteurs traditionnels de la formation (ibid.).

Pour autant, la formation peut constituer un levier d'action essentiel pour développer les compétences de service. Quels sont alors les dispositifs de formation les plus pertinents pour ce type de compétence ? La question est d'autant plus importante que, dans nos deux enquêtes, nous avons constaté que la formation était un vecteur de développement des compétences.

Dans le cas des agents d'ambiance, outre une préparation spécifique des jeunes à la passation des tests classiques de la SNCF pour leur permettre d'intégrer à terme les métiers statutaires, il a été décidé, dans la région de Paris Sud-Est, de faire de la formation un espace de capitalisation des pratiques professionnelles et des compétences acquises in situ (Collard et Iserentant, 2000). Les formateurs ont ainsi encouragé les retours réflexifs sur les situations de travail. Il s'agissait de faire en sorte que les agents prennent progressivement conscience de leurs compétences, qu’ils identifient et qu’ils « déconstruisent » collectivement les savoir-faire implicites mobilisés dans leur travail.

Lors de la seconde enquête, nous avons relevé l'existence de dispositifs de formation innovants mis en place sous l'égide de l'Université du service de la SNCF, afin de développer les compétences de service des agents d'escale. Les ateliers d'improvisation théâtrale, les mises en situation professionnelles et les jeux de rôle filmés, l'utilisation de la PNL (programmation neuro-linguistique) et de l'analyse transactionnelle pour " relire " les situations évoquées spontanément par les agents dans le cadre de la formation, l'identification par les agents de situation-types récurrentes et problématiques, la prise en compte de «l'intermétier " (pour permettre aux agents d'avoir une meilleure vision de la chaîne de service), ou encore le coaching des encadrants de proximité pour développer leur sens de l'observation, constituent quelques-unes de ces innovations. 
Ces démarches, expérimentées dans le cadre du lancement du TGV Est en 2006, restent cependant peu développées au regard des formations traditionnelles (comme les « formations métier »), plus prescriptives et normatives, et des démarches managériales fondées sur une logique de contrôle des comportements, comme les enquêtes client mystère (Collard, 2013).

\subsection{Quels leviers d'action pour reconnaître et développer les compétences de service?}

La formation peut donc être un levier incontournable pour développer ces compétences de service, à condition de partir des situations de travail vécues par les agents sur le terrain et d'opter pour des pédagogies qui stimulent la réflexion des apprenants.

La formation constitue donc un vecteur de développement des compétences dans la mesure où elle est au fondement d'un mouvement qui va de la non-conscience initiale à la prise de conscience, des "compétences incorporées" (Leplat, 1997) aux "compétences explicitées" (Pastré, 1994, 2001)5.

Reconnaitre et développer les compétences de service, via les outils d'évaluation notamment, s'avère en revanche beaucoup plus problématique.

D'une part, parce que les compétences qui figurent dans ces outils correspondent rarement aux compétences mobilisées par les agents en situation de travail ${ }^{6}$. C'est en tout cas ce qui ressort de nos enquêtes à la SNCF. Nos études de cas confortent ainsi les analyses de Baroin-Loos (2003), d'Ughetto (2002) et de Beauquier (2003) pour qui les référentiels de compétences véhiculent des représentations réductrices des compétences de service.

D'autre part, parce que les évaluations réalisées par l'encadrement de proximité risquent de reposer sur des bases tronquées et générer de l'amertume et du ressentiment chez les agents au contact avec la clientèle. Si doter les managers d'outils leur permettant d'évaluer leurs agents sur des bases " objectives " apparaît comme une intention louable, cela n'est pas sans risque. En effet, alors même que ces outils sont censés mettre en lumière les compétences des agents, ils risquent d'aboutir à l'effet inverse de celui recherché. Dès lors que les situations complexes ainsi que les compétences mobilisées dans ces situations ne sont pas répertoriées, cela n’incite pas les managers à les évaluer. C'est peut-être là une des limites fortes du "modèle de la compétence " (Zarifian, 2001).

5. La dynamique évoquée par Pastré renvoie à la prise de conscience telle que Piaget (1974) la décrit, c'està-dire au "passage d'une coordination agie (la dimension incorporée des compétences) à une coordination conceptuelle de l'action" (Pastré, 2001, p. 7).

6. On retrouve ici la tension quasiment irréductible entre le "travail prescrit " et le "travail réel ", bien documentée par les ergonomes. 
D'où l'importance, selon nous, (au-delà du débat sur le bien-fondé des outils de GRH), du type de relation qui s'instaure au sein des équipes entre les managers et leurs agents, mais également entre les agents.

Dans un en-deçà de la formation, par exemple lors des échanges entre agents à la cantine (Gamble, 2006), on trouve des espaces d'apprentissage et de développement des compétences qui sont d'autant plus importants que les formations innovantes mentionnées peuvent n'être que ponctuelles ou en tout cas fragiles.

Prendre en compte le " travail réel » et ses dilemmes pour les agents, en faire un objet de discussion, susciter des dynamiques collectives d'apprentissage en mettant en place des dispositifs ouverts (ex : réunions d'équipe, débriefings, retours d'expérience, etc.) et en encourageant les partages d'expérience informels, constituent quelques exemples de bonnes pratiques pour développer un management " sensible ", attentif aux résistances et aux ruses du réel, capable de reconnaître et de développer les compétences de service des agents de contact.

\section{Conclusion}

Dans son article introductif à un dossier sur les compétences de service, Korczynski (2005) conclut en s'interrogeant sur la possibilité de développer et de mieux reconnaitre ces compétences via le jeu du marché (des services et du travail), les politiques $\mathrm{RH}$, l'action publique et l'action syndicale. De son point de vue, la difficulté à mesurer de telles compétences ("soft»), la faiblesse de l'appartenance syndicale, ou encore la disponibilité de travailleurs peu coûteux apparemment déjà pourvus de ces compétences (par exemple les étudiants), constituent autant de facteurs qui concourent à un diagnostic pessimiste.

Nos résultats s'inscrivent dans cette optique, moins pour souligner la difficulté de l'action à un niveau macro sur les compétences étudiées que pour interroger les outils de gestion des compétences et proposer un ensemble d'actions attentives au contexte.

En matière de gestion des compétences, Gilbert et Le Boulaire (2011) distinguent deux approches. La première, qui a pour objectif de concevoir des outils permettant de répertorier des compétences et de les gérer ${ }^{7}$, tend à " décontextualiser » les compétences et leur gestion. La seconde, qui a pour finalité d'apprendre et de développer des compétences individuelles et collectives, met l'accent sur le contexte organisationnel, toujours spécifique, dans lequel se déploient les compétences.

Nous optons résolument pour la seconde approche. Les compétences de service, par leur nature, nous semblent nécessiter des outils qui prennent en compte le contexte dans lequel elles se déploient. Les formations, quand elles sont en prise avec le travail réel, peuvent alors constituer un levier d'action pertinent.

7. On retrouve ici les outils classiques de la GPEC, comme les référentiels de compétences. 
Par ailleurs, le repérage des espaces informels au sein desquels les agents partagent leurs pratiques et les mettent en discussion, de même que l'identification des dynamiques collectives d'apprentissage impulsées par l'encadrement de proximité (par exemple dans le cadre des retours d'expérience), constituent d'autres pistes fructueuses.

D'où l'intérêt de repérer à la fois les interstices organisationnels et les pratiques des managers de proximité les plus aptes à développer et reconnaître les compétences implicites de service des agents. A cet égard, les démarches et les pratiques les plus intéressantes ne semblent pas passer prioritairement par l'explicitation et l'objectivation de ces compétences...

Quid alors des démarches plus traditionnelles de gestion des compétences comme la GPEC ? Nous butons ici sur une difficulté majeure. En effet, la reconnaissance institutionnelle des compétences professionnelles passe nécessairement par les outils de GRH classiques, ne serait-ce, par exemple, que pour gérer les carrières, organiser les mobilités, ou encore rémunérer les personnes. Autant d'actes de gestion qui reposent sur des critères institutionnels et objectivés. Mais, dans le même temps, ils s'avèrent de bien mauvais alliés pour développer les compétences de service et susciter des dynamiques d'apprentissage au sein des équipes. Ce n'est pas le moindre des paradoxes...

\section{Bibliographie}

Alis D. (1998), "Relation de service : des compétences à développer, des conflits à gérer ", Education Permanente, $\mathrm{n}^{\circ}$ 137, pp. 71-81.

Alis D. et M. Thévenet (1994), "Pour une gestion des ressources humaines adaptée au marketing des services ", Revue Française de Gestion, n ${ }^{\circ} 98$, pp. 119-123.

Bailly F. et Léné A. (2013), “The personification of the service labour process and the rise of soft skills: a French case study”, Employee Relations, vol. 35, n¹, pp. $79-97$.

Baroin-Loos J. (2003), « Les mécanismes de production des compétences dans l'entreprise orientée client ", Economies et sociétés, Série " Economie et gestion des services ", n ${ }^{\circ}$, pp. 1919-1938.

Beauquier S. (2003), Enjeux, contraintes et potentialités des organisations "orientées clients" - le cas de deux entreprises de service : ASSUR et la RATP, Thèse de doctorat de l'Ecole Nationale des Ponts et Chaussées, Marne-la-Vallée.

Bellier S. (2000), Compétences en action, expérimentations, implications, réflexions pratiques, Éditions Liaisons.

Bernard J. (2007), "La gestion des émotions aux pompes funèbres, une compétence reconnue ?", Formation Emploi, n ${ }^{\circ} 99$, pp. 61-74. 
Borzeix A. (1995), "Qualité et bienveillance : l'épreuve de l'étranger ", in Joseph I., Jeannot G. (dir.), Métiers du public : les compétences de l'agent et l'espace de l'usager, pp. 87-123, CNRS Editions, Paris.

Borzeix A. et Collard D. (1999), « La "gestion” des gares de banlieue est-elle une compétence ?", Education Permanente, n ${ }^{\circ} 141$, pp. 83-96.

Borzeix A., Collard D., Raulet-Croset N., Teulier R. (2008), Les compétences implicites dans la relation de service à la $S N C F$, Rapport de recherche réalisé pour le compte de la SNCF, PREG-CRG de l'Ecole Polytechnique.

Boujut S. (2005), « Le travail social comme relation de service ou la gestion des émotions comme compétence professionnelle ", Déviance et Société, vol. 29, n 2, pp. 141-153.

Bourdieu P. (1998), Contre-feux, Liber, Paris.

Caroly S. et Trompette P. (2006), « De la compétence de service aux compétences de coordination et d'orchestration : autour du conseiller funéraire ", Pistes, vol. $8, \mathrm{n}^{\circ} 1$.

Collard D. et Iserentant K. (2000), « Compétences acquises en situation de travail et formation ", Actualité de la formation permanente, $\mathrm{n}^{\circ}$ 167, pp. 79-84.

Collard D. (2003), «La médiation : une compétence ingérable ? ", Gérer et Comprendre, $\mathrm{n}^{\circ} 72$, pp. 15-25.

Collard D. et Raulet-Croset N. (2006), " La dynamique de l'apprentissage "situé". L'exemple des compétences dites de "médiation" ", Revue de Gestion des Ressources Humaines, ${ }^{\circ}$ 59, pp. 17-30.

Collard D. (2013), «Les enquêtes «client mystère " : une technique de contrôle des comportements ", Revue Politiques et Management Public, vol. 30, $\mathrm{n}^{\circ}$ 4, pp. 519-535.

Collard D., Raulet-Croset N., Teulier R. et Suquet J.-B. (2013), "Les managers de proximité face aux compétences : une approche située ", Gérer et Comprendre, n 113 , pp. 62-72.

Combes M.-C. (2002), "La compétence relationnelle : une question d'organisation », Travail et Emploi, $\mathrm{n}^{\circ}$ 92, pp. 5-18.

Commissariat Général au Plan (2001), Services : organisation et compétences tournées vers le client - une lecture transversale des contrats d'études prospectives tertiaires, La documentation française, Paris.

Cosnier J. et Picard D. (1992), « La relation de service en station : analyse pragmatique des interactions agents-usagers à la RATP ", Réseaux 2000, n 67, RATP, Paris.

Dartevelle M. (1992), «Le travail du contrôleur. Les activités langagières des agents », Les Annales de la Recherche Urbaine, n 57-58, pp. 110-114. 
Gadrey J. (1994a), « La modernisation des services professionnels : rationalisation industrielle ou rationalisation professionnelle ? ", Revue Française de Sociologie, vol. 35, n 2, pp. 163-195.

Gadrey J. (1994b), "Les relations de service et l'analyse du travail des agents », Sociologie du Travail, vol. 36, $\mathrm{n}^{\circ}$ 3, pp. 381-389.

Gamble J. (2006), "Multinational Retailers in China: Proliferating 'McJobs' or Developing Skills?”, Journal of Management Studies, vol. ${ }^{\circ} 43$, n 7, pp. 1463-1490.

Gilbert P. et Le Boulaire M. (2011), «De nouvelles voies pour gérer les compétences : priorité au contexte ", Entreprise et personnel, Etude n 295.

Glaser B-G. et Strauss A. (1967), The discovery grounded theory: strategies for qualitative research, Aldine, Chicago.

Goffman E. (1968), Asiles, Les Editions de Minuit, Paris.

Grosjean M. (1995), "Sur quel ton le dîtes-vous ? ", in Joseph I., Jeannot G. (dir.), Métiers du public : les compétences de l'agent et l'espace de l'usager, pp. 159-182, CNRS Editions, Paris.

Havard C. (2006), "Les transformations du travail opérées " au nom du client " : quels défis pour la gestion des ressources humaines?", XVIIème congrès de l'AGRH - Le travail au coeur de la GRH, Reims.

Hochschild A. (1983), The Managed Heart : The Commercialization of Human Feeling, University of California Press, Berkeley, CA.

Honoré L. (2002), "Rupture stratégique, changement organisationnel et rôle de la GRH », Revue de Gestion des Ressources Humaines, n 45, pp. 55-69.

Joseph I. et Jeannot G. (1995), Métiers du public : les compétences de l'agent et l'espace de l'usager, pp. 185-191, CNRS Editions, Paris.

Korczynski M. (2005), "Skills in service work: an overview”, Human Resource Management Journal, 2005, vol. 15, n 2, pp. 3-14.

Krohmer C. (2006), "Les régulations, un mode d'approche du processus de la compétence collective ", XVIIème congrès de l'AGRH - Le travail au cour de la GRH, Reims.

Le Boterf (1994), De la compétence. Essai sur un attracteur étrange, Editions d'Organisation, Paris.

Le Breton E. (1999), "L'utilisateur des transports collectifs urbains : une identité en débat au sein des entreprises ", Sociologie du Travail, vol. 41, n 3, pp. 255-273.

Leplat J. (1997), Regards sur l'activité en situation de travail, PUF, Paris.

MacClelland C. (1961), The Achieving Society, Van Nostrand, Princeton, NJ. 
MacDonald C. L. \& Merrill D. (2009), "Intersectionality in the emotional proletariat", in Korczynski M. \& MacDonald C. L. (Eds.), Service work: Critical perspectives. New York: Routledge.

Mayen P. (2005), "Travail de relation de service, compétences et formation ", in Cerf M., Falzon P. (dir.), Situations de service : travailler dans l'interaction, pp. 61-83, PUF, Paris.

Mitrani A., Murray M. et Bernard A. (1992), Des compétences et des hommes. Le management des ressources humaines en Europe, Editions d'Organisation, Paris.

Pastré P. (1994), "Le rôle des schèmes et des concepts dans la formation des compétences ", Performances Humaines et Techniques, n 71, pp. 21-28.

Pastré P. (2001), « Les compétences professionnelles et leur développement ", La revue de la CFDT, n³9, pp. 3-10.

Payne J. (2009), "Emotional Labour and Skill: A Reappraisal”, Gender, Work and Organization, vol. 16, $\mathrm{n}^{\circ} 3$, pp. 348-367.

Piaget J. (1974), Réussir et comprendre, PUF, Paris.

Strobel P. (1994), "Service public et relation de service: de l'usager au citoyen ", in De Bandt J., Gadrey J. (dir.), Relations de services, marchés de service, pp. 43-62, CNRS Editions, Paris.

Tannery F. (2001), « Le management stratégique des services : synthèse bibliographique et repérage de questions génériques ", Finance Contrôle Stratégie, vol. 4, n² 2, pp. 215-259.

Ughetto P. (2002), "Compétence de service : état des lieux d'une problématique ", Document de travail de l'IRES, $\mathrm{n}^{\circ} 02 / 03$.

Valléry G. (2004), « Relations de service et approche ergonomique : saisir le caractère situé et dynamique de l'activité à travers l'analyse des interactions agent-client ", Activités, vol. 1, n 2, pp. 121-146.

Värlander S., Julien A. (2010), "The effect of the Internet on front-line employee skills: exploring banking in Sweden and France", The Service Industries Journal, vol. 30, $\mathrm{n}^{\circ} 8$, pp. 1245-1261.

Weller J.-M. (1997), "Le guichet interactif : ce que font les bureaucrates lorsqu'ils répondent au téléphone ", Réseaux, n 82-83, pp. 129-148.

Weller J.-M. (1999), « Les compétences de l'agent de bureau dépendent aussi de son guichet ", Education Permanente, n 141, pp. 69-82.

Zarifian P. (2001), Le modèle de la compétence, Editions Liaisons, Paris. 